\title{
Evaluation of Vegetative Parameters of Exotic Pomegranate (Punica granatum L.) Germplasms under Mid-Hill Zones of Himachal Pradesh, India
}

\author{
Suparna Sinha ${ }^{1 *}$, Ankita Aman ${ }^{1}$, Jyoti Kumari ${ }^{1}$, Kajal Kiran ${ }^{2}$ and Ruby Rani ${ }^{1}$ \\ ${ }^{1}$ Department of Horticulture (Fruit and Fruit Tech.), ${ }^{2}$ Departmentof Soil Science and \\ Agricultural Chemistry, Bihar Agricultural University, Sabour, Bhagalpur-813210, India \\ *Corresponding author
}

\section{A B S T R A C T}

\begin{tabular}{|l|}
\hline Ke y w or d s \\
$\begin{array}{l}\text { Pomegranate, Yield, } \\
\text { Mid-Hill conditions }\end{array}$ \\
\hline Article Info \\
\hline $\begin{array}{l}\text { Accepted: } \\
\text { 23 March } 2018 \\
\text { Available Online: } \\
\text { 10 April } 2018\end{array}$ \\
\hline
\end{tabular}

\section{Introduction}

In India pomegranate production traditionally more under arid zone conditions. Considering the increasing population, expanding pomegranate cultivation in non-traditional areas may contribute towards nutritional security. The experimental farmat an altitude of 1270 meters above mean sea level lying between $35.5^{\circ}$ North latitude and $77.8^{\circ}$ East longitude. The farm area falls in the mid-hill zone of Himachal Pradesh. Most of the farmers in this zone are interested to cultivate temperate horticultural crops (like apple, peach, pear, plum etc.) for their remunerative return. The pomegranate is known to be dry land horticultural crops. Hence, an attempt was made for the collection of exotic pomegranate varieties for identifying a suitable germplasm to the mid-hill zone which can replace temperate horticultural crops to improve the financial conditions of farmers as well as mitigate climate effect with creating varietal diversification.

Twenty germplasms (Table 1) were evaluated for testing their performance in mid hill conditions. The growth parameters viz., plant height (m), plant spread (m), trunk girth (mm), number of stems, number of suckers, thorn length and length of internodes were recorded in all germplasm. 
Table.1 Plant growth characteristics of twenty different pomegranate germplasms

\begin{tabular}{|c|c|c|c|c|c|c|c|c|c|c|c|}
\hline \multirow{2}{*}{\multicolumn{2}{|c|}{$\begin{array}{l}\text { Germplasm } \\
\text { accessions }\end{array}$}} & \multirow[t]{2}{*}{$\begin{array}{l}\text { Growth } \\
\text { habit }\end{array}$} & \multirow[t]{2}{*}{$\begin{array}{l}\text { Heigh } \\
\text { t (m) }\end{array}$} & \multicolumn{2}{|c|}{$\begin{array}{l}\text { Plant spread } \\
\text { (m) }\end{array}$} & \multirow{2}{*}{$\begin{array}{l}\text { Trunk } \\
\text { girth } \\
(\mathbf{m m})\end{array}$} & \multirow{2}{*}{$\begin{array}{c}\text { Numbe } \\
\text { r of } \\
\text { stems }\end{array}$} & \multirow{2}{*}{$\begin{array}{c}\text { Number } \\
\text { of } \\
\text { suckers }\end{array}$} & \multirow{2}{*}{$\begin{array}{l}\text { Thorn } \\
\text { length } \\
(\mathrm{mm})\end{array}$} & \multirow{2}{*}{$\begin{array}{l}\text { Length } \\
\text { of } \\
\text { internod } \\
\text { es (cm) }\end{array}$} & \multirow[t]{2}{*}{ Colour of young shoot } \\
\hline & & & & N-S & E-W & & & & & & \\
\hline 1. & 20090265 & Spreading & 1.35 & 1.19 & 0.58 & 40.34 & 1.00 & 2.00 & 8.26 & 2.91 & Red Group 46 C \\
\hline 2. & $\begin{array}{l}\text { AlkPustGhern } \\
\text { ezSveh }\end{array}$ & Spreading & 1.68 & 0.55 & 0.49 & 38.78 & 1.33 & 0.67 & 0.00 & 2.43 & Red Group 50 A \\
\hline 3. & Al-sirin-nar & Spreading & 1.85 & 1.46 & 1.16 & 44.71 & 1.00 & 8.67 & 6.52 & 2.65 & Red Group 50 B \\
\hline 4. & Cloud & Upright & 1.59 & 0.45 & 0.44 & 60.44 & 2.00 & 1.00 & 8.55 & 3.08 & Red Group 53 B \\
\hline 5. & Crab & Spreading & 2.15 & 1.66 & 1.69 & 88.19 & 2.33 & 5.67 & 6.52 & 3.24 & Red Group 50 B \\
\hline 6. & Dewey & Spreading & 3.18 & 1.61 & 1.74 & 96.09 & 2.00 & 5.67 & 7.12 & 2.85 & Red Group 51A \\
\hline 7. & Eve & Spreading & 2.19 & 1.97 & 1.47 & 21.21 & 1.67 & 0.67 & 5.13 & 2.64 & Red Group 48 B \\
\hline 8. & Green Globe & Spreading & 3.16 & 2.54 & 2.54 & 49.84 & 2.00 & 1.00 & 5.67 & 4.32 & Red Group 50 A \\
\hline 9. & Gulyalek & Spreading & 2.33 & 2.08 & 2.13 & 65.91 & 2.33 & 28.67 & 6.59 & 2.87 & Red Group 52 A \\
\hline 10. & Haku-botan & Spreading & 2.10 & 1.61 & 1.26 & 65.30 & 3.33 & 0.67 & 7.18 & 3.34 & $\begin{array}{l}\text { Yellow Green Group } \\
144 \text { D }\end{array}$ \\
\hline 11. & Kaim-anar & Upright & 1.65 & 0.46 & 0.38 & 28.20 & 2.00 & 0.67 & 9.21 & 3.25 & Red Group 51A \\
\hline 12. & Loulou & Upright & 3.09 & 2.65 & 2.489 & 91.11 & 3.00 & 5.00 & 9.58 & 2.62 & Red Group 52 A \\
\hline 13. & Nusai & Upright & 2.18 & 1.55 & 1.54 & 51.16 & 1.67 & 1.00 & 9.96 & 3.26 & Red Grroup 51 B \\
\hline 14. & Orange & Spreading & 2.15 & 1.35 & 1.77 & 35.50 & 2.33 & 0.67 & 10.00 & 2.76 & Red Group 51 C \\
\hline 15. & Ovadan & Spreading & 1.62 & 0.88 & 1.14 & 32.76 & 2.00 & 2.67 & 9.18 & 3.72 & Red Group 52 A \\
\hline 16. & Parfyanets & Drooping & 2.31 & 1.65 & 1.88 & 40.98 & 3.00 & 3.00 & 6.85 & 2.80 & Red Group 48 B \\
\hline 17. & Podarok & Upright & 2.37 & 1.57 & 1.69 & 38.54 & 1.67 & 1.00 & 7.82 & 3.23 & Red Group 51 A \\
\hline 18. & Purple Heart & Upright & 1.55 & 0.54 & 0.34 & 20.11 & 1.00 & 0.67 & 0.00 & 2.03 & Red Group $51 \mathrm{C}$ \\
\hline 19. & Saharnyi & Spreading & 2.24 & 2.20 & 1.57 & 59.00 & 2.33 & 7.00 & 6.24 & 3.55 & Red Group 48 B \\
\hline 20. & Sogdiana & Upright & 1.82 & 1.62 & 1.38 & 24.30 & 3.33 & 0.67 & 6.13 & 3.09 & Red Group 39 A \\
\hline \multicolumn{2}{|c|}{ Mean } & & 2.13 & 1.48 & 1.38 & 49.62 & 2.02 & 3.85 & 6.82 & 3.03 & \\
\hline \multicolumn{2}{|c|}{ C. $D_{0.05}$} & & $0.14^{*}$ & $0.09 *$ & $0.05 *$ & $0.18 *$ & $0.84^{*}$ & $0.73^{*}$ & $0.04^{*}$ & $0.05 *$ & \\
\hline
\end{tabular}


Table.2 Foliage characteristics of twenty different pomegranate germplasms

\begin{tabular}{|c|c|c|c|c|c|c|c|c|c|c|c|}
\hline \multirow{2}{*}{\multicolumn{2}{|c|}{ Germplasm accessions }} & \multirow{3}{*}{$\begin{array}{c}\begin{array}{c}\text { Date of } \\
\text { leaf bud } \\
\text { break }\end{array} \\
21 / 2 / 13 \\
\end{array}$} & \multirow{3}{*}{$\begin{array}{c}\begin{array}{c}\text { Leaf } \\
\text { Length } \\
(\mathbf{m m})\end{array} \\
43.50\end{array}$} & \multirow{3}{*}{$\begin{array}{c}\begin{array}{c}\text { Leaf } \\
\text { Breadth } \\
(\mathbf{m m})\end{array} \\
14.24\end{array}$} & \multirow{3}{*}{$\begin{array}{r}\begin{array}{r}\text { Leaf } \\
\text { area } \\
\left(\mathbf{c m}^{2}\right)\end{array} \\
5.44\end{array}$} & \multirow{3}{*}{$\begin{array}{c}\begin{array}{c}\text { Petiole } \\
\text { length } \\
\text { (mm) }\end{array} \\
5.02\end{array}$} & \multirow{2}{*}{\multicolumn{2}{|c|}{ Colour of leaves }} & \multirow{3}{*}{$\begin{array}{l}\text { Leaf shape } \\
\text { Lanceolate }\end{array}$} & \multirow{3}{*}{$\begin{array}{r}\begin{array}{r}\text { Leaf } \\
\text { apex }\end{array} \\
\text { Acute }\end{array}$} & \multirow{3}{*}{$\begin{array}{l}\begin{array}{l}\text { Time of } \\
\text { leaf fall }\end{array} \\
13 / 11 / 12\end{array}$} \\
\hline & & & & & & & & & & & \\
\hline 1. & 20090265 & & & & & & Greyed Purple Group 187B & Green Group 143A & & & \\
\hline 2. & $\begin{array}{l}\text { AlkPustGherme } \\
\text { zSaveh }\end{array}$ & $22 / 2 / 13$ & 60.39 & 16.03 & 17.97 & 5.18 & Greyed Purple Group 148A & $\begin{array}{l}\text { Yellow Green Group } \\
\text { 147A }\end{array}$ & Lanceolate & Acute & $11 / 12 / 12 *$ \\
\hline 3. & Al-sirin-nar & $21 / 2 / 13$ & 55.54 & 15.16 & 8.24 & 4.88 & Greyed Red Group 181A & Green Group 144B & Lanceolate & Acute & $22 / 11 / 12$ \\
\hline 4. & Cloud & $26 / 2 / 13$ & 96.43 & 19.79 & 20.28 & 9.31 & Greyed Purple Group 187B & Green Group 143A & Lanceolate & Acute & 24/11/12 \\
\hline 5. & Crab & $21 / 2 / 13$ & 83.10 & 19.30 & 20.14 & 5.78 & Greyed Red Group 181A & Green Group 141A & Lanceolate & Acute & 20/11/12 \\
\hline 6. & Dewey & $22 / 2 / 13$ & 53.80 & 17.34 & 13.1 & 4.70 & Greyed Purple Group 183A & Green Group 143A & Lanceolate & Acute & $21 / 11 / 12$ \\
\hline 7. & Eve & $19 / 2 / 13$ & 36.53 & 7.57 & 6.13 & 4.48 & Greyed Purple Group 184A & Green Group 137A & Lanceolate & Acute & 19/11/12 \\
\hline 8. & Green Globe & $22 / 2 / 13$ & 73.70 & 21.50 & 27.75 & 10.01 & Greyed Purple Group 184A & Green Group 143A & Lanceolate & Acute & 21/11/12 \\
\hline 9. & Gulyalek & $22 / 2 / 13$ & 79.64 & 21.68 & 14.12 & 5.42 & Greyed Purple Group 185A & Green Group 137A & Lanceolate & Acute & $18 / 11 / 12$ \\
\hline 10. & Haku-botan & $22 / 2 / 13$ & 77.54 & 22.69 & 19.10 & 5.90 & Yellow Green Group 151C & Green Group 139A & Lanceolate & Acute & $16 / 11 / 12$ \\
\hline 11. & Kaim-anar & $24 / 2 / 13$ & 65.82 & 16.37 & 15.26 & 4.4 & Greyed Purple Group 185A & Green Group 144A & Lanceolate & Acute & $25 / 11 / 12$ \\
\hline 12. & Loulou & $21 / 2 / 13$ & 74.92 & 20.96 & 25.17 & 8.75 & Greyed Purple Group 187C & Green Group 141A & Lanceolate & Acute & 2/12/12 \\
\hline 14. & Orange & $19 / 2 / 13$ & 77.76 & 23.74 & 18.92 & 6.92 & Greyed Purple Group 184B & Green Group 143A & Broad elliptic & Acute & $10 / 12 / 12^{*}$ \\
\hline 15. & Ovadan & $22 / 2 / 13$ & 58.11 & 13.48 & 7.85 & 5.46 & Greyed Purple Group 185A & $\begin{array}{l}\text { Yellow Green Group } \\
\text { 145A }\end{array}$ & Elliptic lanceolate & Acute & 19/11/12 \\
\hline 16. & Parfyanets & $19 / 2 / 13$ & 64.55 & 18.53 & 12.69 & 7.89 & Greyed Purple Group 184B & Green Group 143A & Lanceolate & Acute & $17 / 11 / 12$ \\
\hline 17. & Podarok & $28 / 2 / 13$ & 70.46 & 21.40 & 18.96 & 7.40 & Greyed Purple Group 185A & Green Group 144A & Lanceolate & Acute & $20 / 11 / 12$ \\
\hline 18. & Purple Heart & $14 / 2 / 13$ & 53.78 & 16.96 & 13.54 & 3.33 & Greyed Red Group 181A & Green Group 141A & Lanceolate & Acute & $12 / 12 / 12 *$ \\
\hline 19. & Saharnyi & $22 / 2 / 13$ & 59.76 & 17.31 & 14.61 & 4.14 & Greyed Purple Group 187C & Green Group 143A & Lanceolate & Acute & $4 / 12 / 12$ \\
\hline 20. & Sogdiana & $17 / 2 / 13$ & 46.24 & 12.61 & 6.47 & 3.27 & Greyed Purple Group 184A & Green Group 137A & Lanceolate & Acute & $18 / 11 / 12$ \\
\hline Me & & & 65.40 & 17.7 & 15.03 & 5.50 & & & & & \\
\hline CD & & & $0.21^{*}$ & $2.35^{*}$ & 3.86 & 1.10 & & & & & \\
\hline
\end{tabular}


Table.3 Time of maturity, days taken from full bloom to maturity and yield per plant of twenty different pomegranate germplasms

\begin{tabular}{|l|}
\hline Germplasm accessions \\
\hline 20090265 \\
\hline AlkPustGhermezSaveh \\
\hline Al-sirin-nar \\
\hline Cloud \\
\hline Crab \\
\hline Dewey \\
\hline Eve \\
\hline Green Globe \\
\hline Gulyalek \\
\hline Haku-botan \\
\hline Kaim-anaranar \\
\hline Loulou \\
\hline Nusai \\
\hline Orange \\
\hline Ovadan \\
\hline Parfyanets \\
\hline Podarok \\
\hline Purple Heart \\
\hline Saharnyi \\
\hline Sogdiana \\
\hline Mean \\
\hline CD 05 \\
\hline
\end{tabular}

\begin{tabular}{|c|c|c|}
\hline Time of maturity & $\begin{array}{c}\text { Days from full bloom to } \\
\text { maturity }\end{array}$ & Yield per plant (kg) \\
\hline $12 / 8 / 13$ & 118 & 1.60 \\
\hline $15 / 8 / 13$ & 110 & 1.74 \\
\hline $10 / 8 / 13$ & 110 & 1.99 \\
\hline $18 / 8 / 13$ & 115 & 1.72 \\
\hline $17 / 8 / 13$ & 123 & 3.80 \\
\hline $5 / 8 / 13$ & 110 & 5.60 \\
\hline $8 / 8 / 13$ & 114 & 2.44 \\
\hline $8 / 8 / 13$ & 108 & 13.60 \\
\hline $16 / 8 / 13$ & 112 & 4.90 \\
\hline $15 / 8 / 13$ & 61 & 2.86 \\
\hline $10 / 8 / 13$ & 107 & 1.11 \\
\hline $18 / 8 / 13$ & 124 & 10.9 \\
\hline $15 / 8 / 13$ & 124 & 5.58 \\
\hline $7 / 8 / 13$ & 118 & 2.63 \\
\hline $24 / 8 / 13$ & 130 & 2.03 \\
\hline $19 / 8 / 13$ & 126 & 4.34 \\
\hline $17 / 8 / 13$ & 117 & 3.51 \\
\hline $10 / 8 / 13$ & 119 & 1.40 \\
\hline $16 / 8 / 13$ & 118 & 3.30 \\
\hline \multirow[t]{3}{*}{$8 / 8 / 13$} & 118 & 1.88 \\
\hline & & 3.85 \\
\hline & & $0.02 *$ \\
\hline
\end{tabular}


The plant were planted during 2009 in an RBD design with 20 treatments and 3 replications. The data were collected during 2013 and their average mean values were taken into consideration.

Among the twenty pomegranate germplasms studied, Dewey $(3.18 \mathrm{~m})$ was highest in plant height which was at par with Green Globe $(3.16 \mathrm{~m})$ and Loulou $(3.09 \mathrm{~m})$. Plant height, plant spread (N-S and E-W) and cumulative stem girth was found to vary between 1.35 $3.18 \mathrm{~m}, 0.45-2.65 \mathrm{~m}(\mathrm{~N}-\mathrm{S})$ and $0.34-2.54$ $\mathrm{m}(\mathrm{E}-\mathrm{W})$ and $20.11-.96 .09 \mathrm{~mm}$, respectively suggesting considerable variation in plant vigour. Such variations in growth characters are reported by Sharma and Bist (2005). The maximum internodal length was observed in Green Globe $(4.32 \mathrm{~cm})$ with the overall mean for intermodal length was recorded as 3.03 $\mathrm{cm}$. The leaf area $\left(27.75 \mathrm{~cm}^{2}\right)$ and fruit yield/tree $(13.6 \mathrm{~kg})$ were recorded highest (Table 2 and 3) in Green Globe followed by Loulou (leaf area $25.17 \mathrm{~cm}^{2}$ and yield 10.9 $\mathrm{kg}$ ). Wani et al., (2012) reported that leaf area of wild pomegranate selections accessions ranged between $7.48 \mathrm{~cm}^{2}$ and $14.04 \mathrm{~cm}^{2}$. Also, Rao and Subramanyam (2009) reported that among four varieties of pomegranate nine years old Mridula recorded with highest fruit yield/tree $(14.1 \mathrm{~kg})$ under scarce rainfall zone. Days from bloom to maturity ranged from 61 days (Haku-botan) to 130 days (Ovadan). Though, duration from anthesis to maturity in pomegranate has been reported to vary from three to five months (Patil and Karale, 1992).

The present studies do indicate that some of these germplasms (like Dewey, Green Globe, Loulou) do possess one or more horticulturally desirable characteristics and thus can be future commercial varieties provided they yield quality fruits consistently and sufficiently.

\section{References}

Patil A V and Karale A R. 1992. Pomegranate. In: Fruits of India. Tropical and sub-tropical. Eds. Bose, T. K. and Mitra S K. Naya Prokash, Calcutta. pp. 537-548.

Rao K D and Subramanyam K. 2009. Evaluation of yield parameters of pomegranate varieties under scarce rainfall zone. Agic. Sci. Digest. 29(2): 12.

Sharma N and Bist H S. 2005. Evaluation of some pomegranate cultivars under mid hills of Himachal Pradesh. Acta Horticulture. 696: 103-105.

Wani I A, Bhat M Y, Lone AA, Banday F A, Khan I A and GanaiShaiq A. 2012. Variation in some promising selections of wild pomegranate (Punica granatum L.) in central Kashmir. Applied Biological Research. 14(2): 211-214.

\section{How to cite this article:}

Suparna Sinha, Ankita Aman, Jyoti Kumari, Kajal Kiran and Ruby Rani. 2018. Evaluation of Vegetative Parameters of Exotic Pomegranate (Punica granatum L.) Germplasms under MidHill Zones of Himachal Pradesh, India. Int.J.Curr.Microbiol.App.Sci. 7(04): 3079-3083. doi: https://doi.org/10.20546/ijcmas.2018.704.349 